\title{
Intelligent Nutrition in Healthcare and Continuous Care
}

\author{
Rui Miranda \\ Algoritmi Research Centre \\ University of Minho \\ Braga, Portugal \\ rui@ruimiranda.dev
}

\author{
Diana Ferreira \\ Algoritmi Research Centre \\ University of Minho \\ Braga, Portugal \\ a72226@alunos.uminho.pt
}

\author{
António Abelha \\ Algoritmi Research Centre \\ University of Minho \\ Braga, Portugal \\ abelha@di.uminho.pt
}

\author{
José Machado \\ Algoritmi Research Centre \\ University of Minho \\ Braga, Portugal \\ jmac@di.uminho.pt
}

\begin{abstract}
In the healthcare industry, the patient's nutrition is a key factor in their treatment process. Every user has their own specific nutritional needs and requirements. An appropriate nutrition policy can therefore help the patient's recovery process and alleviate possible symptoms.

Food recommender systems are platforms that offer personalised suggestions of recipes to users. However, there is a lack of usage of recipe recommendation systems in the healthcare sector. Multiple challenges in representing the domain of food and the patient's needs make it complicated to implement these systems.

The present project aims to develop a platform for an intelligent planning of the user's meals, based on their clinical conditions. The application of machine learning algorithms on nutrition, in healthcare services and continuous care is thus a key topic of research. This platform will be tested and deployed at the Social Canteen of Vila Verde (Cantina Social da Santa Casa da Misericórdia de Vila Verde).

The development of this project will use the Design Science Research (DSR) investigation methodology, ensuring that the solution to the problem accomplishes all needs and requirements of the professionals, while elucidating new knowledge both for the institution and the scientific community.

Index Terms-machine learning, recommender systems, meal planning, decision support systems
\end{abstract}

\section{INTRODUCTION}

One of the biggest hurdles in healthcare is decision making, as every option has its advantages and possible complications, which may jeopardise the human life. A recent solution to this problem is the implementation of Decision Support Systems (DSSs). When applied to healthcare, these systems can improve the patient's quality of life, offering personalised and patient-focused suggestions [1], [2].

The patient's nutrition is a key component in its treatment and recovery process. Medical nutrition therapy, also called nutrition therapy, use nutrition as a treatment process. A person's nutrition status is checked, and given the right foods or nutrient to treat conditions, such as those caused by diabetes, heart disease, and cancer [3], [4]. A medical nutrition therapy policy may also help patients recover more quickly, spending less time in the hospital [5].

In this context, this project has emerged, consisting in the development and exploration of a new platform for meal planning, incorporating machine learning algorithms for decision support, helping the health professionals in the Social Canteen of Vila Verde. In particular, this system encompasses a web application, where all meals at the Santa Casa da Misericórdia de Vila Verde and at the hospital of Vila Verde can be reserved for later preparation, and a mobile app, where individuals at the refectory can order their meals, see the menu of the week, and offer feedback regarding a dish.

A key feature in this platform is the suggestion of food menus to each department, taking into consideration the patient's nutritional needs. Data regarding the patient's nutritional requirements will be gathered and analysed, as well as feedback from the department's coordinators and the refectory's users. Therefore, a food recommender system will be developed for decision support, helping the Social Canteen's planning.

Accordingly, the main motivation for this project focuses on developing and exploring a new generation of tools in the field of Information Technologies (ITs), in order to help with the meal planning and management for the multiple divisions offered to the community by the Santa Casa da Misericórdia de Vila Verde.

This way, both web and mobile applications will be developed, using several methodologies and technologies currently available and viable for the conception of the defined IT solutions, also called IT artefacts. A data retrieval of the canteen's operations will enable the creation of machine learning models for the food plan recommendation system.

The presented paper is organised as follows: section I presents a contextualisation and framing of the work as well as its main objectives; section II presents theoretical and scientific concepts of interest, namely machine learning and recommender systems; section III presents the Design Science Research (DSR) investigation methodology adopted for the development of the platform; section IV refers to the discussion of the results achieved; section $\mathrm{V}$ presents the main conclusions obtained thorough the development of the platform, as well as showcasing future work in order to improve the system.

\section{A. Objectives}

The main objective of the present study is the development and exploration of a new platform for meal planning, incorporating machine learning algorithms for decision support, helping the health professionals in the Social Canteen of Vila 
Verde. This platform is comprised of two main tools, one for simplifying the Social Canteen's operations, namely a web platform for the provision of meal plans, which contains a food recommender module for decision support of the food plans, incorporating machine learning algorithms, and a mobile application for the refectory's users. Accordingly, the following objectives were highlighted:

- Requirements elicitation in the design and development of the platform;

- Analysis of the available methodologies and technologies involved in the design and development of the system;

- Creation of a web application in React with the following purposes:

- Development of an improved meal reservation system, where all coordinators can easily order meals for their departments;

- Creation of dashboards, allowing an overview of the canteen's operations, and any other possible indicators of service;

- Integration with the hospital's acquisition system, allowing a seamless food ordering process.

- Creation of a mobile application in React Native, where the users can easily obtain the menu and effortlessly order food trough their smartphones;

- Development of a recommender system for the suggestion of food menus using machine learning approaches for its automatic creation, taking into consideration the patient's nutritional needs and requirements;

- Implementation, deployment and maintenance of both tools in the Social Canteen of Vila Verde.

\section{StATE OF THE ART}

In this section, several topics of interest to the project are studied and documented. A careful analysis of past papers and articles allows to understand what other investigators and researchers have previously done, their challenges, and how they were able to overcome them.

\section{A. Machine Learning in Nutrition}

Machine learning is a field of study that focuses on the ability for computer systems to "learn" from data and identify patterns with minimal human intervention [6]-[8].

Tom Mitchell had offered a definition for the ability for computer software to learn as: "A computer program is said to learn from experience $E$ with respect to some class of tasks $T$ and performance measure $P$, if its performance at tasks in $T$, as measured by $P$, improves with experience $E$." $[9,2]$. Machine learning uses advanced statistical techniques and algorithms to improve the performance measure, allowing computer systems to learn from experience.

Countless articles and thesis are based on machine learning systems, making an impact in computation, prediction and automation. In addition, data mining studies employ the same methods as machine learning for extracting knowledge. In literature, several healthcare and nutrition projects have implemented machine learning systems, employing a diverse cast of algorithms.

R. Reis, H. Peixoto, J. Machado, and A. Abelha have implemented a data mining study, by combining a nutritional dataset with machine learning techniques. Their main objective was to predict if a patient would need to be followed by a nutrition specialist. The CRoss Industry Standard Process for Data Mining (CRISP-DM) methodology was used, as well as data extracted from a Portuguese hospital. Five machine learning models were included, namely Decision Trees, Support Vector Machines Bayesian Networks, Decision Rules and Nearest Neighbours. They were able to create multiple models, discovering which attributes were able to predict the need for a nutrition specialist, helping decision-makers to take the best decision, predicting events before their occurrence [10].

D. Zeevi et al. have devised a machine learning algorithm for the prediction of personalised postprandial (post-meal) glycemic responses. The system integrates blood parameters, dietary habits, anthropometrics, physical activity and gut microbiota, for a total of 137 attributes. A cohort of 800 participants was used to gather data, validating the model with another cohort of 100 participants. This study has also performed a blinded randomised controlled dietary intervention based on this algorithm, resulting in lower postprandial responses and alterations to gut microbiota [11].

J. Aravind and J. D. Sweetlin have developed a system for nutrient facts analysis, using machine learning systems. To make nutrition facts labels more accessible and easier to understand, they applied supervised learning approaches to classify food products by their level of healthiness. Multiple models were tested and compared, including K-Nearest Neighbours, Support Vector Machines, and Classification and Regression Trees (CARTs) obtaining a maximum accuracy of 73 [12].

\section{B. Food Recommender Systems}

Recommender Systems are defined as "(...) software tools and techniques that provide suggestions for items that are most likely of interest to a particular user." $[13,1]$.

Its main ability is to provide personalised and relevant recommendations to the users. Recommender systems focus on a specific domain of items (for example, movies, books or music), the user's graphical interface, and the recommendation techniques used to generate useful suggestions for that type of item $[13,1]$.

Food recommender systems offer suggestions of recipes and/or food items to the users, taking into consideration their wishes and needs. Nowadays, modern food recommender systems not only recommend recipes suiting user's preferences, but also suggest healthier choices. Food recommender systems can also keep track of the eating behaviour of the user and help understand the impact of nutrition in its health, persuading the user's eating behaviour.

Food recommender systems can be developed for multiple target audiences and goals. These systems can be classified in several types: the ones that consider the user's preferences, 
recommending healthier recipes or food items similar to the ones the user liked in the past; the ones that consider the nutritional needs of the users, recommending items that have been identified beforehand by health care providers; the ones that strike a balance between the user's preferences and its nutritional needs; if they recommend food for groups, in which food items are consumed by groups of users rather than by individuals [14, 507].

Existing research on food recommender systems is summarised and compared in how they take into account the user's preferences and/or their nutritional needs, and how the system generates those suggestions.

T. De Pessemier, S. Dooms, and L. Martens have proposed a food recommender for patients in a care facility. As many of those patients cannot express their personal preferences, a recommender system can assist in the selection of the menu items that match a patient's preferences. A framework was also developed to store data about preferences, activities, and behaviour of patients regarding meals .

In addition, the framework developed can also monitor the amount of food eaten by every patient over time, detecting irregularities in the patient's eating behaviour. Caregivers can use this information to keep track of the patient's health condition [15].

G. Agapito et al. have presented a recommender system for the adaptive delivery of nutrition contents, improving the quality of life of both healthy subjects and patients with dietrelated chronic diseases. The system also contains catalogues of typical regional foods from Calambria, characterised by their nutraceutical properties.

To give users dietary advice, the system bases on the user's health status, the disease's data, and the nutritional information of the food. The system evaluates whether a food item is compatible with the user's health status, and sorts according to how much appropriate they are. However, the system does not suggest based on the user's preferences. Instead, the system uses a knowledge-based approach for food recommendation [16].

C.-Y. Teng, Y.-R. Lin, and L. A. Adamic seek to distil the collective knowledge and preferences about cooking, using data mining algorithms on a popular recipe-sharing website. To extract information, the unstructured text of recipes and the accompanying user reviews are parsed.

Two types of networks were constructed, one reflecting different relationships between ingredients and other to capture the user's knowledge on how to combine ingredients. The authors performed a prediction task, where given a pair of similar recipes, the algorithm should determine which one has a higher average rating than the other. Discriminative machine learning algorithms, such as support vector machines and stochastic gradient boosting trees were used for this prediction problem [17].

\section{RESEARCH StRATEGIES}

The development of this project will base upon the Design Science Research (DSR) investigation methodology used on the construction and evaluation of reliable and rigorous IT solutions. Additionally, in every phase of the system development, from the data mining process to the final prototype, multiple methodologies, technologies and toolkits considered appropriate for the definition and implementation of each component of the final solution were adopted and implemented.

Design Science Research can be described as a "research paradigm in which a designer answers questions relevant to human problems via the creation of innovative artefacts, thereby contributing new knowledge to the body of scientific evidence" [18, 5].

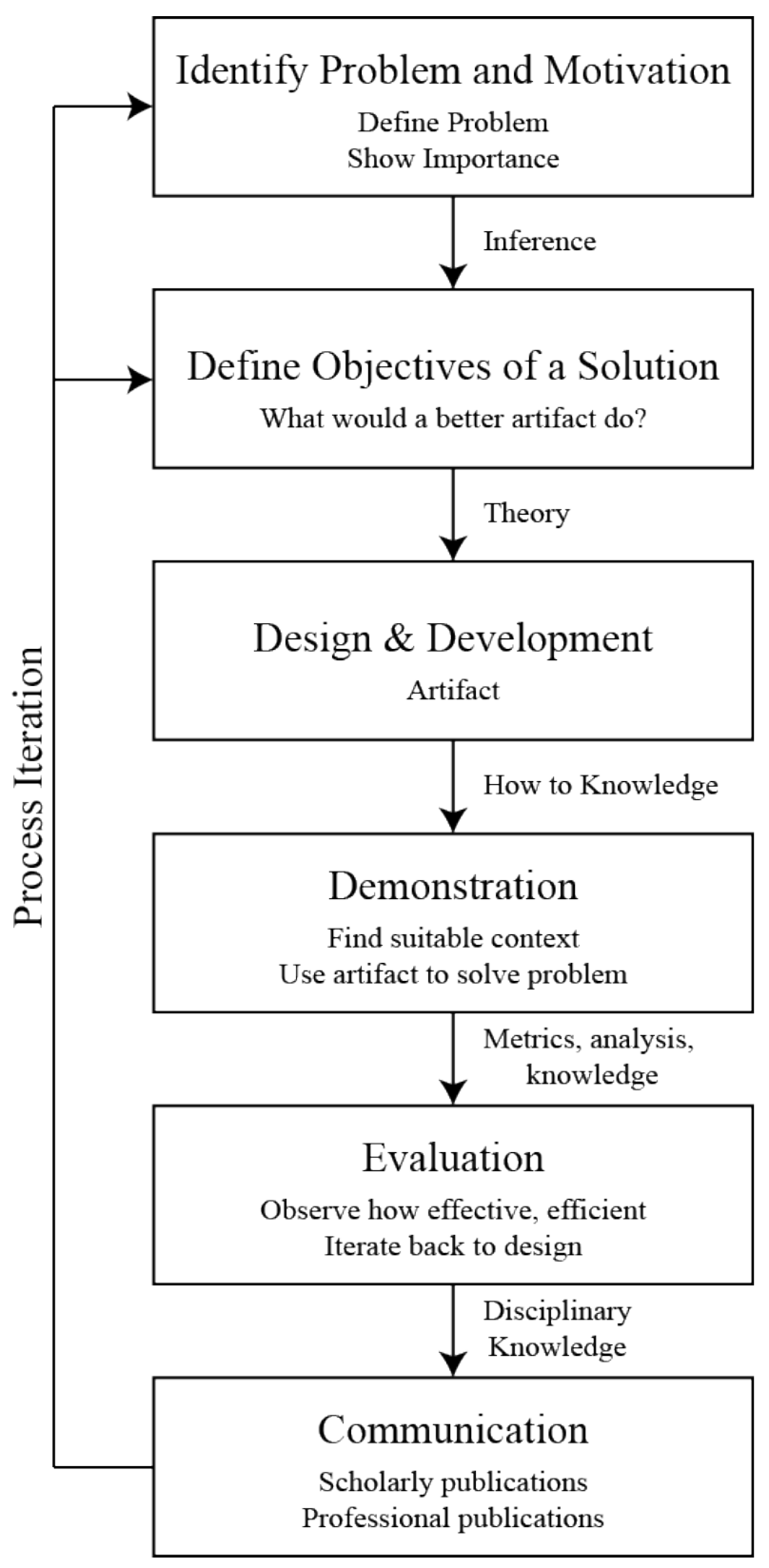

Fig. 1. Design Science Research Methodology [19, 15].

The initial phase of the Design Science Research (DSR) methodology is the awareness of the problem, its definition 
and the assessment of its importance, outputting a proposal for a new research effort.

Afterwards, a solution must be presented with a proper foundation, where new functionalities are envisioned based on a novel configuration of either existing or new elements, and with the benefits that the new artefact could accomplish.

The new artefact is then developed and evaluated according to implicit criteria made explicit in the proposal. If the artefact confirms a hypothesis, it can be considered as the final solution, that may be shared or published.

This methodology uses an iterative approach, allowing the freedom to adapt the structure and the evaluation of the developed artefact, until an ideal solution is found [20, 8-10].

\section{RESUlTS AND DisCUSSION}

In this section, the architecture of the platform and the adopted technologies, as well as the advances in representing and storing key information are presented. A Strengths Weaknesses Opportunities Threats (SWOT) analysis was performed, identifying the strengths, weaknesses, opportunities and threats to help develop a strong strategic plan.

\section{A. Results}

1) Tools and Architecture: To successfully develop the system, multiple tools and technologies were studied and the most appropriate were chosen to create the solution.

The chosen technologies are:

1) Node.js, an open-source, cross-platform JavaScript runtime environment used to create the web server. In addition, the Express framework is used to build the system's web services, and Passport.js to ensure a secure authentication process;

2) JSON Web Tokens (JWTs) (JWT) is used for authorisation, allowing the users to access routes and resources;

3) MySQL is the Database Management System (DBMS), storing all information regarding users and business requirements;

4) React is a JavaScript library used to build the interface of the web application;

5) React Native is a software developer kit used to develop an Android and iOS application using the same codebase;

The project uses a client-server architecture. Figure 2 represents the platform's architecture, its main components and interactions.

2) Data Representation: This platform requires the storage of information concerning the users, menus, dishes, ingredients and their nutritional information. The MySQL Database Management System (DBMS) stores all tables and relationships, while the Sequelize Object-Relational Mapping (ORM) is used to define all models and interact with the database.

Multiple key tables have been defined and modelled:

- Dish: represents all information regarding dishes. A dish is made up of multiple preparations, allowing the reuse of

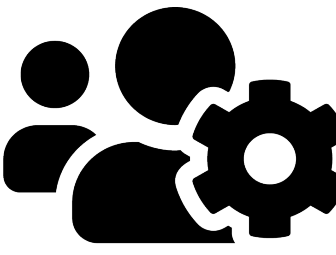

Social Canteen
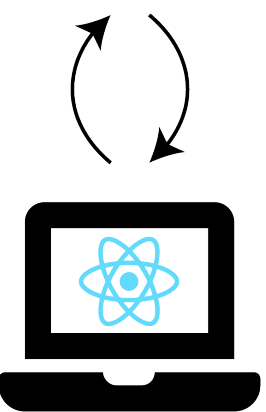

Front-end

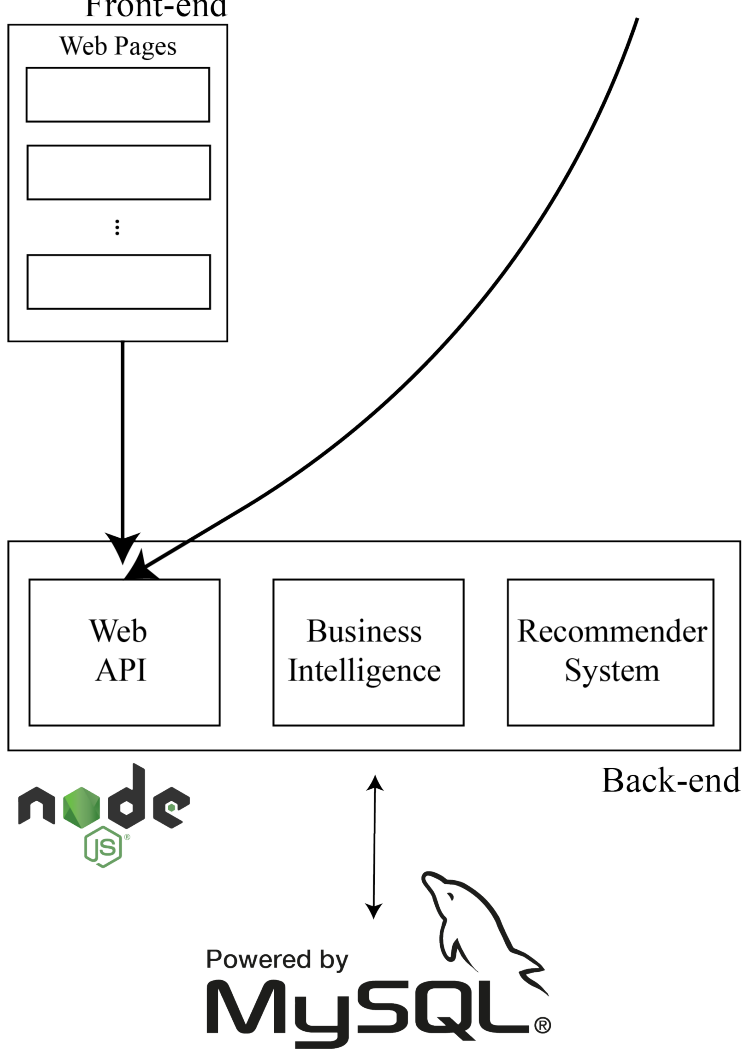

Fig. 2. System Architecture and Tools.
Refectory User
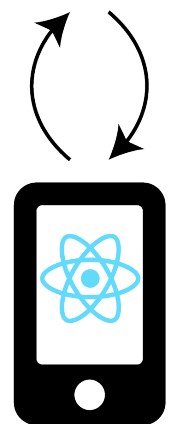

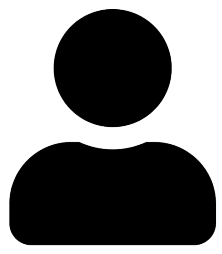


common preparations, simplifying the process of creating dishes.

- Preparation: represents all information regarding preparations. A preparation is comprised of several ingredients, and cooking instructions.

- Ingredient: represents all information regarding ingredients. An ingredient is made up of nutritional components per potion, potential allergens, and an edible part percentage. The ingredient's nutritional information was obtained from PortFIR, a Portuguese food information platform, containing 1206 food products [21].

- Menu: stores all menus, the dishes chosen for each division, and how many users are expected.

- User: represents all registered users, user type, and interactions with the platform.

\section{B. Discussion}

SWOT analysis is a planning technique used to identify the strengths and weaknesses (internal factors), in addition to opportunities and threats (external factors) of a business or project plan [22]. A SWOT analysis can help reveal opportunities to exploit, understand potential weaknesses, and develop strong strategic plans [23], [24]. The SWOT analysis enabled the identification of the following:

- Strengths

- Development of a high quality, easy to use platform;

- Increased efficiency and compliance with the Hazard Analysis and Critical Control Point (HACCP) system;

- New interactions with the social canteen and its customers and patients;

- Usage of machine learning approaches for nutrition therapy.

- Weaknesses

- Machine learning algorithms may suggest ill-suited dishes;

- Transition to a new interface may require training;

- Persistent connection to the internet is required.

- Opportunities

- New departments within the Santa Casa da Miserdicória de Vila Verde may offer new possibilities;

- Mobile app allows the exploration of new interactive features;

- Recent trends for the user's health and nutrition awareness.

- Threats

- Complex laws and regulations (GDPR, HACCP,...);

- Complex architecture, requiring the maintenance of a back-end and two front-end applications;

- New data may require the development of a new machine learning based approach.

\section{CONCLUSion ANd Future Work}

Information technology systems are changing the healthcare sector, from the discovery of cures for diseases and the development of new treatment techniques to the improvement of patient's diagnosis and the enhancement of effective health care delivery. From health centres to large scale hospitals, the increased use of IT approaches in clinical acts is improving the patient's quality of care, as well as optimising the health institution's resources [25].

The nutrition status of the patient is crucial for his/her treatment process. Food recommender systems provide personalised recommendations of food items and/or recipes to the users and may take into consideration the health status of the user improving its health status. Although there are several food recommender systems in the literature, health institutions fail to implement and use such systems. The present study aims to analyse the specific needs of a health institution in order to understand what functionalities and requirements a nutritional recommendation system must have in order to be successfully implemented and used by a health institution. This project results from the collaboration of the department of informatics of the Minho University, in Braga, with a Portuguese hospital, the Santa Casa da Misericórdia de Vila Verde. Currently, the project counts on the collaboration of a work team made up of programmers and health professionals, with the ability to intervene and participate in the multiple phases of the project.

Through the methodologies and investigation strategies chosen, it was possible to delineate a valid strategy, starting from topics and key ideas that, with the revision of the literature, became more solid and justified. Accordingly, the end result of this project was the creation of a new approach for meal planning, using the patient's preferences and nutritional needs for decision support. Thus, a new intelligent system on nutrition in healthcare and continuous care was developed, based on machine learning algorithms, using data mining to identify patterns and improve the patient's quality of care.

As future work, in the upcoming months, the proposed system will be evaluated and implemented at the Social Canteen of Vila Verde, in order to detect errors or inconsistencies boosting its continuous improvement and maintenance.

\section{ACKNOWLEDGEMENTS}

\section{REFERENCES}

[1] J. Neves, H. Vicente, M. Esteves, F. Ferraz, A. Abelha, J. Machado, J. Machado, J. Neves, J. Ribeiro, and L. Sampaio, "A Deep-Big Data Approach to Health Care in the AI Age," Mobile Networks and Applications, vol. 23, no. 4, pp. 1123-1128, 8 2018. [Online]. Available: http://link.springer.com/10.1007/s11036-018-1071-6

[2] M. Esteves, A. Abelha, and J. Machado, "The development of a pervasive Web application to alert patients based on business intelligence clinical indicators: a case study in a health institution," Wireless Networks, 1 2019. [Online]. Available: https://doi.org/10.1007/ s11276-018-01911-6

[3] NCI Dictionary of Cancer Terms, "Definition of medical nutrition therapy - NCI Dictionary of Cancer Terms - National Cancer Institute,' p. 1, 2018. [Online]. Available: https://www.cancer.gov/publications/ dictionaries/cancer-terms/def/medical-nutrition-therapy

[4] S. F. Morris and J. Wylie-Rosett, "Medical nutrition therapy: A key to diabetes management and prevention," Clinical Diabetes, vol. 28, no. 1, pp. $12-18,2010$. 
[5] D. Ferreira, H. Peixoto, J. Machado, and A. Abelha, "Predictive Data Mining in Nutrition Therapy," in 2018 13th APCA International Conference on Control and Soft Computing (CONTROLO). IEEE, 6 2018, pp. 137-142. [Online]. Available: https://ieeexplore.ieee.org/ document/8516413/

[6] Wikipedia contributors, "Machine learning - Wikipedia, The Free Encyclopedia," 2018. [Online]. Available: https://en.wikipedia.org/w/ index.php?title=Machine_learning\&oldid=868337401

[7] R. Kohavi and F. Provost, "Glossary of Terms Journal of Machine Learning," 1998. [Online]. Available: http://ai.stanford.edu/ ronnyk/ glossary.html

[8] "Machine Learning: What it is and why it matters," p. 1, 2018. [Online]. Available: https://www.sas.com/en_gb/insights/analytics/ machine-learning.html

[9] T. M. Mitchell, Machine Learning, 1st ed., McGraw-Hill, Ed. New York, NY, USA: McGraw-Hill, Inc., 1997.

[10] R. Reis, H. Peixoto, J. Machado, and A. Abelha, "Machine Learning in Nutritional Follow-up Research,” Open Computer Science, vol. 7, no. 1, pp. 41-45, 12 2017. [Online]. Available: http://www.degruyter.com/ view/j/comp.2017.7.issue-1/comp-2017-0008/comp-2017-0008.xml

[11] D. Zeevi, T. Korem, N. Zmora, Z. Halpern, E. Elinav, E. S Correspondence, D. Israeli, D. Rothschild, A. Weinberger, O. BenYacov, D. Lador, T. Avnit-Sagi, M. Lotan-Pompan, J. Suez, J. A. Mahdi, E. Matot, G. Malka, N. Kosower, M. Rein, G. Zilberman-Schapira, L. Dohnalová, M. Pevsner-Fischer, R. Bikovsky, E. Segal, Z. Halpern, E. Elinav, and E. Segal, "Personalized Nutrition by Prediction of Glycemic Responses," Cell, vol. 163, no. 5, pp. 1079-1095, 2015. [Online]. Available: http://dx.doi.org/10.1016/j.cell.2015.11.001

[12] J. Aravind and J. D. Sweetlin, "Nutrient facts analysis using supervised learning approaches," in 2017 Conference on Information and Communication Technology, CICT 2017, vol. 2018-April, 2018, pp. 1-6.

[13] N. Rubens, M. Elahi, M. Sugiyama, and D. Kaplan, Recommender Systems Handbook, F. Ricci, L. Rokach, and B. Shapira, Eds. Boston, MA: Springer US, 2015, vol. 247, no. 6403. [Online]. Available: http://link.springer.com/10.1007/978-1-4899-7637-6

[14] T. N. Trang Tran, M. Atas, A. Felfernig, and M. Stettinger, "An overview of recommender systems in the healthy food domain," Journal of Intelligent Information Systems, vol. 50, no. 3, pp. 501-526, 6 2018. [Online]. Available: http://link.springer.com/10.1007/ s10844-017-0469-0

[15] T. De Pessemier, S. Dooms, and L. Martens, "A food recommender for patients in a care facility," Proceedings of the 7th ACM conference on Recommender systems - RecSys '13, pp. 209-212, 2013. [Online]. Available: http://dl.acm.org/citation.cfm?doid=2507157.2507198

[16] G. Agapito, M. Simeoni, B. Calabrese, I. Caré, T. Lamprinoudi, P. H. Guzzi, A. Pujia, G. Fuiano, and M. Cannataro, "DIETOS: A dietary recommender system for chronic diseases monitoring and management," Computer Methods and Programs in Biomedicine, vol. 153, pp. 93-104, 2014. [Online]. Available: https://doi.org/10.1016/j.cmpb.2017.10.014

[17] C.-Y. Teng, Y.-R. Lin, and L. A. Adamic, "Recipe recommendation using ingredient networks," in Proceedings of the 3rd Annual ACM Web Science Conference on - WebSci '12, vol. V-59-09. New York, New York, USA: ACM Press, 2012, pp. 298-307. [Online]. Available: https://arxiv.org/pdf/1111.3919.pdf?utm_source=www.mazavr.tk\&utm medium=referralhttp://dl.acm.org/citation.cfm?doid=2380718.2380757

[18] A. R. Hevner and S. Chatterjee, Design Research in Information Systems: Theory and Practice. Springer, New York, NY, 2010, vol. 2. [Online]. Available: http://link.springer.com/10.1007/ 978-1-4419-6108-2

[19] K. Peffers, T. Tuunanen, M. A. Rothenberger, and S. Chatterjee, "A Design Science Research Methodology for Information Systems Research,” Journal of Management Information Systems, vol. 24, no. 3 pp. 45-77, 12 2007. [Online]. Available: https://www.tandfonline.com/ doi/full/10.2753/MIS0742-1222240302

[20] B. Kuechler and S. Petter, "Design Science Research in Information Systems," Design Science Research in Information Systems:, pp. 1-66, 2017. [Online]. Available: http://desrist.org/ desrist/content/design-science-research-in-information-systems.pdfhttp: //www.desrist.org/design-research-in-information-systems/

[21] Instituto Nacional de Saúde Dr. Ricardo Jorge, "Tabela da Composição de Alimentos," 2016. [Online]. Available: http://portfir.insa.pt

[22] W. Kenton, "SWOT Analysis Definition," 2019. [Online]. Available: https://www.investopedia.com/terms/s/swot.asp
[23] Wikipedia contributors, "SWOT analysis," 2019. [Online]. Available: https://en.wikipedia.org/w/index.php?title=SWOT_analysis\& oldid $=885318472$

[24] Mind Tools Content Team, "SWOT Analysis (Strengths, Weaknesses Opportunities, Threats)," p. 1, 2019. [Online]. Available: https: //www.mindtools.com/pages/article/newTMC_05.htm

[25] L. Cardoso, F. Marins, F. Portela, M. Santos, A. Abelha, and J. Machado, "The next generation of interoperability agents in healthcare." International journal of environmental research and public health, vol. 11, no. 5, pp. 5349-71, 5 2014. [Online]. Available: http://www.ncbi.nlm.nih.gov/pubmed/24840351http://www. pubmedcentral.nih.gov/articlerender.fcgi?artid=PMC4053905 